\title{
Normal EEG
}

\author{
William O. Tatum, IV
}

T

he EEG is a unique and valuable measure of the brain's electrical function. It is a graphic display of a difference in voltages from two sites of brain function recorded over time. EEG involves the study of recording these electrical signals that are generated by the brain. Extracranial EEG provides a broad survey of the electrocerebral activity throughout both hemispheres of the brain. Intracranial EEG provides focused EEG recording directly from the brain from surgically implanted electrodes that are targeted at specific regions of the brain. Information about a diffuse or focal cerebral dysfunction, the presence of interictal epileptiform discharges (IEDs), or patterns of special significance may be revealed. For the successful interpretation of an abnormal EEG, one must first understand the criteria necessary to define normal patterns. While a normal EEG does not exclude a clinical diagnosis of epilepsy, an abnormal finding on EEG may be supportive of the clinical diagnosis. Furthermore, it may be indicative of cerebral dysfunction by demonstrating focal or generalized slowing. The results of a standard EEG may have nothing to do with the reason that the study was performed (i.e., patients with headache) and the results need to be taken in context with the individual reason for referral. It is the clinical correlation applied to the findings of the EEG that imparts its utility.

Electrical signals are created when electrical charges move within the central nervous system. Neural function is normally maintained by ionic gradients established by neuronal membranes. Sufficient duration and length of small amplitude potentials (measured in microvolts) of electrical currents generated by cerebral activity are required to be amplified and displayed for visual interpretation. Normally, a resting (diffusion) membrane potential exists through the efflux of positive-charged (potassium) ions maintaining electro-chemical equilibrium at $-75 \mathrm{mV}$. With depolarization, an influx of positive-charged (sodium) ions exceeding the normal electrochemical resting potential occurs. Ion channels open within the lipid bilayer via a voltage-dependent mechanism, and closure is timedependent. Conducting the change in cellular depolarization to adjacent portions of the nerve cell membranes results in an action potential when threshold is exceeded. However, it is the synaptic potentials as opposed to the action potentials that are the most important source of the extracellular current flow to produce the EEG. Excitatory postsynaptic potentials (EPP) flow inwardly (extracellular to intracellular) to other parts of the cell (sinks), via sodium or calcium ions. Inhibitory postsynaptic potentials (IPP) flow outwardly (intracellular to extracellular) in the opposite direction (source) and involve chloride or potassium ions. These summed potentials are longer in duration than action potentials and are responsible for most of the EEG waveforms. The brainstem and thalamus serve as subcortical generators to synchronize populations of neocortical neurons in both normal (i.e., sleep elements) and in abnormal situations (i.e., generalized spike-and-wave complexes). Volume conduction characterizes the process of current flow from the brain generator and recording electrode.

Layers of cortical neurons are the main source of the EEG. Pyramidal cells are the major contributor of the synaptic potentials that make up EEG (Figure 2.1A). These neurons are arranged in a perpendicular orientation to the cortical surface from layers III, IV, and VI. 
Volumes large enough to permit measurement at the surface of the scalp require areas that are $>6 \mathrm{~cm}^{2}$ though $>10 \mathrm{~cm}^{2}$ for recording most IEDs on the scalp using the International 10-20 system of electrode placement due to the attenuating properties incurred by the scalp and skull. All generators have both a positive and negative pole and function as a dipole (see Figure 2.1B). The EEG displays the continuously changing voltage fields at different locations on the scalp.

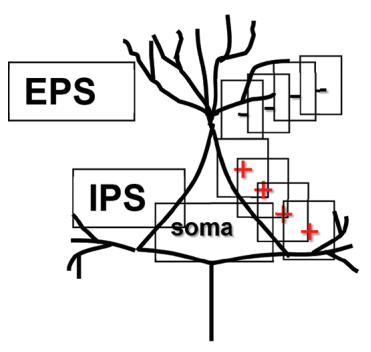

(A)



(B)
FIGURE 2.1. (A) A pyramidal cell with excitatory postsynaptic potentials and inhibitory postsynaptic potentials.

(B) Dipole depicting a field of charge separation.

S tandard EEG recording displays the difference in electrical potentials between two different sites on the scalp overlying cerebral cortex that is closest to the recording electrode (Figure 2.1). During routine use, electrical potentials are acquired indirectly from the scalp surface, and incorporate waveform analyses of frequency, voltage, morphology, and topography. However, most of the human cortex is buried deep beneath the scalp surface. In addition, EEG represents a 2-dimensional projection of a 3-dimensional source. This presents challenges to solve the inverse problem of source localization using scalp EEG. Furthermore, the waveforms that are recorded from the scalp represent pooled synchronous activity from large populations of neurons that create cortical potentials and therefore may not reflect small interictal or ictal generators.

Initial one-channel EEG recordings in the late 1920s have evolved to sophisticated digital-based computerized recording devices. From the patient scalp, electrodes conduct electrical potentials to an electrode box (jackbox). Thereafter, the montage selector permits EEG signals to pass through amplifiers before filtering and ancillary controls regulate the signal output. Data display follows acquisition and processing and has a wide variety of data presentation for EEG interpretation. Electrode placement has been standardized by an international system that uses anatomic landmarks on the skull. These sites are then subdivided by intervals of $10 \%$ and $20 \%$ to designate the site where an electrode will be placed. A minimum of 21 electrodes are commonly used for clinical studies, though digital EEG now has the capability for a greater number and 25 electrodes to accent basal temporal structures are recommended. During infant EEG recordings, fewer electrodes are often used and vary depending upon age and head size. A newer modified combinatorial electrode system uses electrode placement with more closely spaced electrodes in a 10-10 system (see Figure 2.2). The designations, Fp (frontopolar), F (frontal), T (temporal), O (occipital), C (central), and $\mathrm{P}$ (parietal) are utilized in the 10-20 system. Subsequently, numbers combined following the letters for location reflect either the left (odd numbers) or right (even numbers) hemisphere 
of electrode placement. The " $\mathrm{z}$ " designation reflects midline placement (i.e., $\mathrm{Cz}=$ central midline). In the 10-10 system, lower numbers in their positions reflect locations closer to the midline, and T3/T4 become T7/T8 while T5/T6 become P7/P8. Electrode impedances should be maintained between 100 and 5,000 ohms. Special electrodes may also be added, such as sphenoidal, true temporal, or fronto-temporal electrodes. Most are employed for the purpose of delineating temporal localization. True temporal electrodes (designated T1/T2 and FT9/FT10) are placed to help distinguish anterior temporal or posterior inferior frontal location to enhance recovery compared with F7 and F8 electrode positions. Combining the 10-20 system with electrodes from the 10-10 system may be most practical for routine clinical use when additional electrodes are desirable. Collodion is a compound used to secure electrodes during prolonged recording techniques, such as during video-EEG, continuous EEG in the intensive care unit, or ambulatory EEG monitoring. Paste used for routine recordings is more temporary. Subdermal electrodes are used when other recording techniques are not feasible, such as in the OR and ICU.

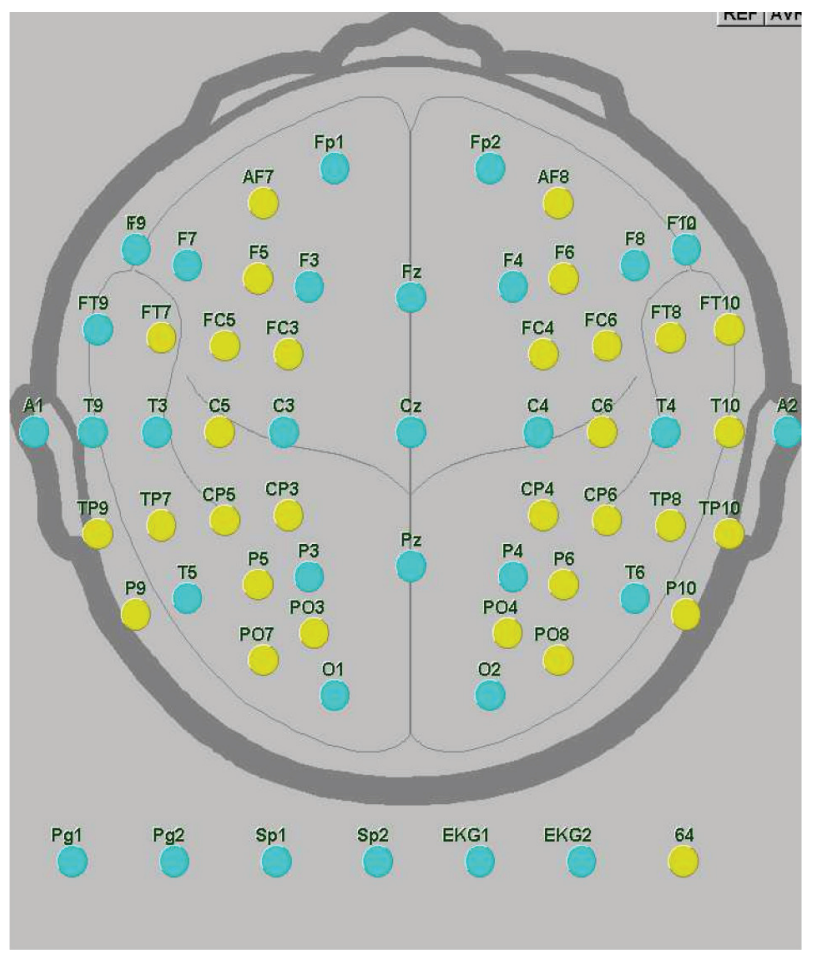

FIGURE 2.2. Electrode placement systems use either a 10-20 system (blue) or modified combinatorial system with 10-10 electrode placement (blue + gold).

O ther additional electrodes may include EKG (recommended with every EEG), eye movement monitors, EMG, and extracerebral electrodes to aid in artifact differentiation (i.e., tremor monitor) and sleep staging. Respiratory monitors may also be important if respiratory problems are identified or sleep monitoring is performed. 




(A)

\section{Fp1-Ref}

F7-Ref

T3-Ref

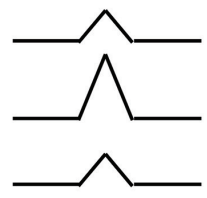

T5-Ref

01-Ref

(B)
FIGURE 2.3. (A) Bipolar montage

demonstrating phase reversal. (B) Referential montage demonstrating absolute voltage.

T he electrical "map" obtained from the spatial array of recording electrodes used to interpret EEG is the montage (Figure 2.3). Several montages are used throughout a 20- to 30-minute EEG recording. Every standard EEG should include at least one montage using an anterior-posterior longitudinal bipolar, reference, and traverse bipolar montage (Figure 2.4). A reference montage uses an active electrode site as the initial input, and then at least one "neutral" electrode to depict absolute voltage. The greatest amplitudes measured are commensurate with the site of maximal electro-negativity or positivity. One midline reference electrode (i.e., Pz), may be useful for a lateralizing temporal recording. However, two references (i.e., ipsilateral ear reference) may be useful for more generalized discharges. Even multiple "averaged" sites of reference (or Laplacian montages for very focal recordings) may be useful for localized discharges. Bipolar montages may be arranged in many different spatial formats, including longitudinally, transverse fashion, or in a circumferential pattern. The longitudinal bipolar (aka "double banana") is the one that is most frequently represented in clinical practice and throughout this text. An anterior to posterior temporal and central transverse connecting chain of electrodes arranged left alternating to rightsided placement is an example of a targeted array to lateralize and cross-localize temporal abnormalities. Bipolar montages compare two active electrodes sites adjacent to each other and signify absolute electrographic sites of maximal negativity (or positivity) by phase-reversals.

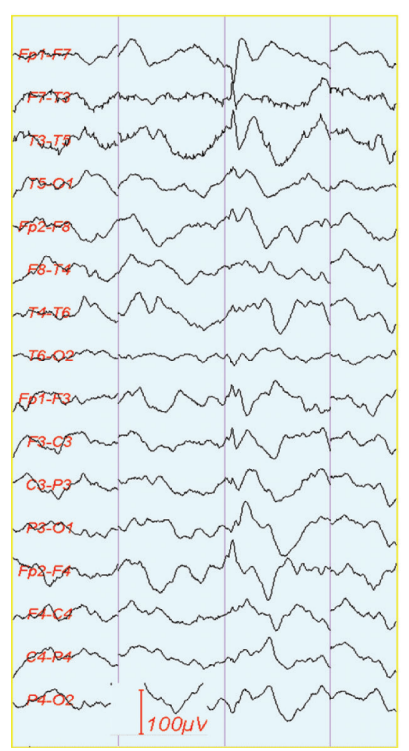

(A)

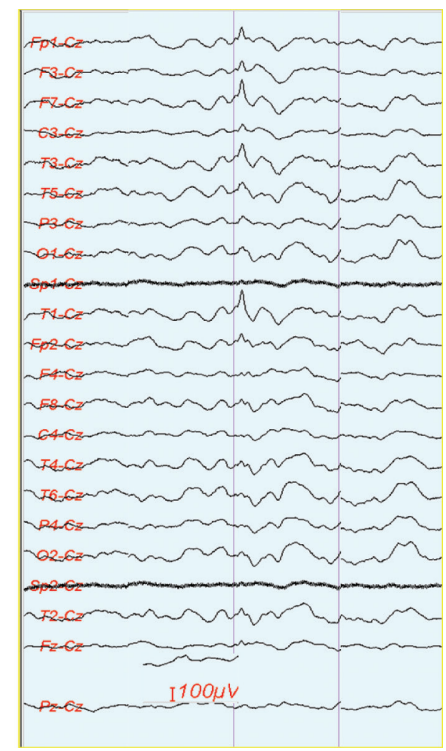

(B)
FIGURE 2.4. EEG demonstrating bipolar (A) and reference (B) montages to illustrate a left anterior temporal sharp wave at the F7 electrode (left) and T1 derivation (right). 


\begin{tabular}{|c|c|c|}
\hline EEG & Electrode 1 & Electrode 2 \\
\hline Negative & Up & Down \\
\hline Positive & Down & Up \\
\hline
\end{tabular}

FIGURE 2.5. The rules governing polarity and convention are relative to deflection of the waveform. By convention, when input 1 is negative, the deflection is up. Polarity of the EEG thus has two reasons for an upward or downward deflection.

y convention, when the voltage difference between electrode 1 is more negative than electrode 2, deflection of the waveform is up (Figure 2.5). Recordings are usually performed with a visual display speed of $30 \mathrm{~mm} / \mathrm{sec}$ (slower with sleep studies). Amplifier sensitivities of $7 \mu \mathrm{V} / \mathrm{mm}$ and filter settings of 1 to $70 \mathrm{~Hz}$ are routine parameters during standard EEG. Reducing the low filter settings promotes slower frequency representation, while reducing high filter settings attenuates high frequencies. A narrow band reduction is possible using a "notched" filter setting to limit $60-\mathrm{Hz}$ interference (or $50 \mathrm{~Hz}$ in some countries outside the United States). Proprietary software offers digital seizure and spike detection capabilities for digital EEG. These systems are commercially available for both standard and prolonged EEG monitoring. This section will encompass patterns of cerebral and extracerebral origin as well as patterns of uncertain significance to illustrate the range of normal EEG encountered in clinical practice. Like most interpreters for clinical EEG, the orderly approach includes (a) review of the parameters of recording, (b) identifying the state of the patient and background activity, and (c) the most salient feature(s) of the epoch. 


\section{NORMAL EEG}

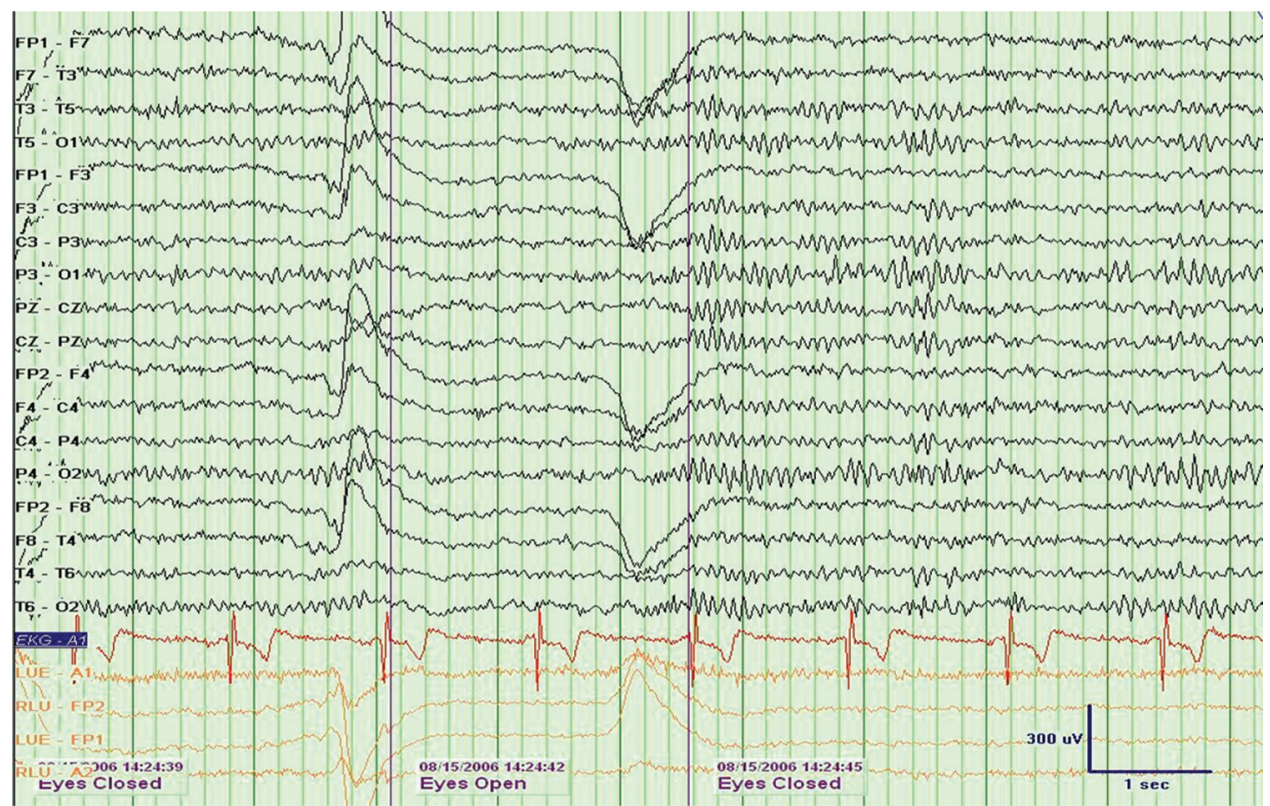

FIGURE 2.6. Normal 10-Hz alpha rhythm "blocked" by eye opening and returning on eye closure. Note the faster frequency immediately on eye closure ("squeak").

$\mathrm{T}$ he alpha rhythm is the starting point in the orderly approach to analyze EEG. In the normal EEG, a posterior dominant rhythm is represented bilaterally over the posterior head regions and lies within the 8- to $13-\mathrm{Hz}$ bandwidth (alpha frequency). When this rhythm is attenuated with eye opening, it is referred to as the alpha rhythm (Figure 2.6). During normal development, an $8-\mathrm{Hz}$ alpha frequency appears by 3 years of age. The alpha rhythm remains stable between 8 and $12 \mathrm{~Hz}$ even during normal aging into the later years of life. In approximately $25 \%$ of normal adults, the alpha rhythm is poorly visualized, and in $<10 \%$, voltages of $<15 \mu \mathrm{V}$ may be seen. The alpha rhythm is distributed maximally in the occipital regions and shifts anteriorly during drowsiness. Voltage asymmetries of $>50 \%$ should be regarded as abnormal especially when the left side is greater than the right. It is best observed during relaxed wakefulness and has a side-to-side difference of $<1 \mathrm{~Hz}$. Unilateral failure of the alpha rhythm to attenuate reflects an ipsilateral abnormality (Bancaud's phenomenon). Normally, alpha frequencies may transiently increase immediately after eye closure (alpha squeak). Alpha variants include forms that are $1 / 2$ (slow alpha) or 2 times (fast alpha) the alpha frequency with similar distribution and reactivity. Alpha variants may have a notched appearance. Paradoxical alpha occurs when alerting results in the appearance of alpha, and drowsiness does not. 


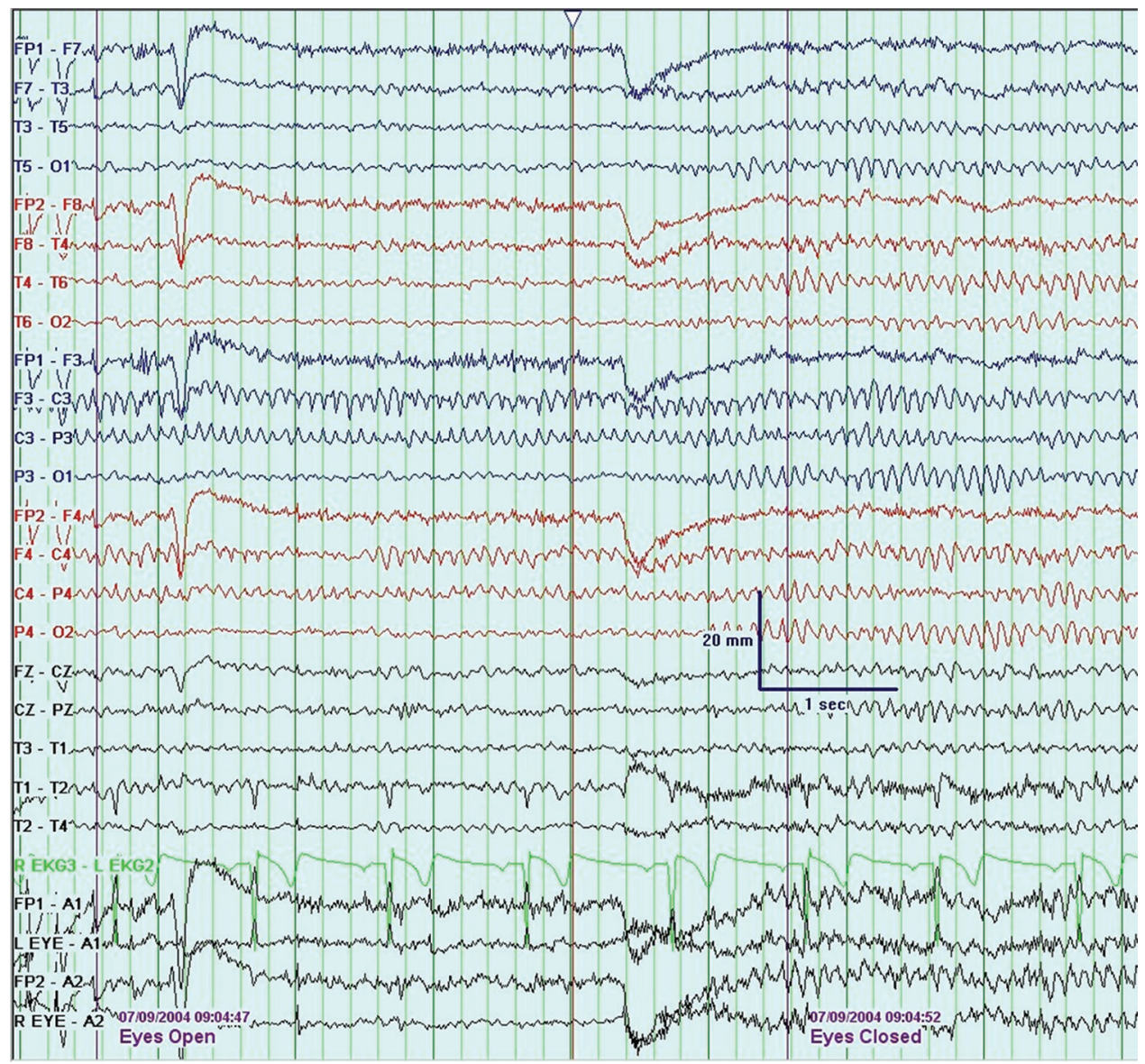

FIGURE 2.7. Note the prominent left central mu rhythm during eye opening.

The $m u$ rhythm is a centrally located arciform alpha frequency (usually 8-10 Hz) that represents the sensorimotor cortex at rest (see Figure 2.7). While it resembles the alpha rhythm, it blocks not with eye opening, but instead with contralateral movement or the thought of making a movement in an extremity. It may be seen only on one side, and may be quite asymmetrical and asynchronous, despite the notable absence of an underlying structural lesion. The $m u$ rhythm may slow with advancing age and is usually of lower amplitude than the existent alpha rhythm. When persistent, unreactive, and associated with focal slowing, $m u$-like frequencies are abnormal. 


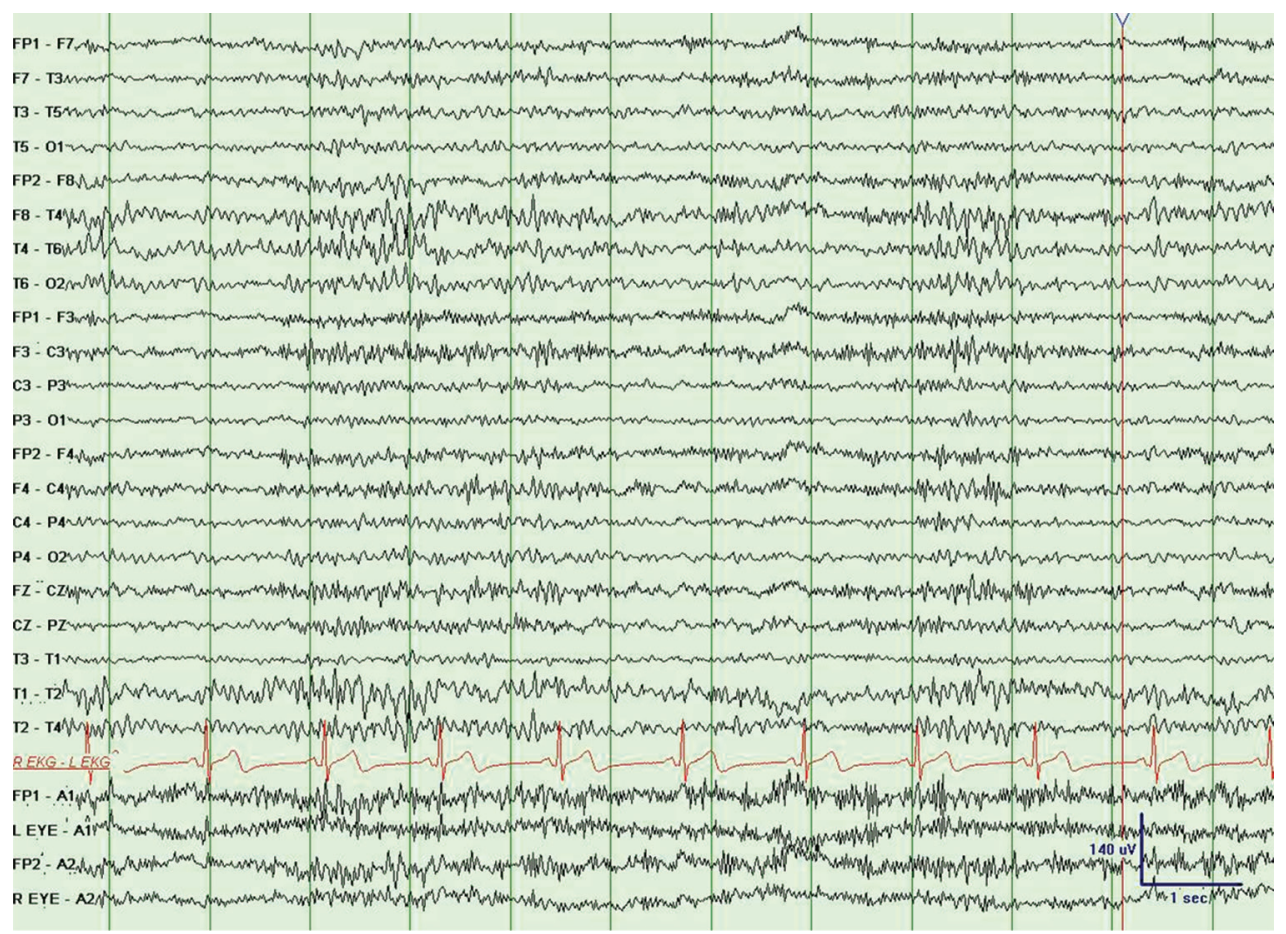

FIGURE 2.8. Breach rhythm in the right temporal region (maximal at T4) following craniotomy for temporal lobectomy.

B eta rhythms are frequencies that are more than $13 \mathrm{~Hz}$. They are common, and normally observed within the 18 - to $25-\mathrm{Hz}$ bandwidth with a voltage of $<20 \mu \mathrm{V}$. Voltages beyond $25 \mu \mathrm{V}$ in amplitude are considered abnormal. Benzodiazepines, barbiturates, and chloral hydrate are potent activators of generalized beta or "fast activity" $>50 \mu \mathrm{V}$ for $>50 \%$ of the waking tracing within the $14-$ to $16-\mathrm{Hz}$ bandwidth. Beta activity normally increases during drowsiness, light sleep, and with mental activation. Persistently reduced voltages of $>50 \%$ suggests a cortical grey matter abnormality within the region or hemisphere having the lower amplitude. However, lesser asymmetries may simply reflect normal skull asymmetries. A skull defect may produce a breach rhythm with focal, asymmetric, higher amplitudes (this relative increase may be $>3$ times). Beta activity is more prominent without the skull to attenuate the faster frequencies. A breach rhythm (Figure 2.8) on the EEG is considered normal for the physiological conditions of recording when it is independent of spikes or focal slowing. 




FIGURE 2.9. Normal frontocentral theta in an 18-year-old while awake.

Theta rhythms are composed of 4 - to 7-Hz frequencies of varying amplitude and morphologies. Approximately one-third of normal awake, young adults show intermittent 6- to $7-\mathrm{Hz}$ theta of $<15 \mu \mathrm{V}$ that is maximal in the frontal or frontocentral head regions (Figure 2.9). The appearance of frontal theta can be facilitated by emotions, focused concentration, and during mental tasks. Theta activity is normally enhanced by hyperventilation, drowsiness, and sleep. Intermittent $4-$ to $5-\mathrm{Hz}$ activity bitemporally, or even with a lateralized predominance (usually left greater than right), may occur in about a third of the asymptomatic elderly and is not abnormal. 


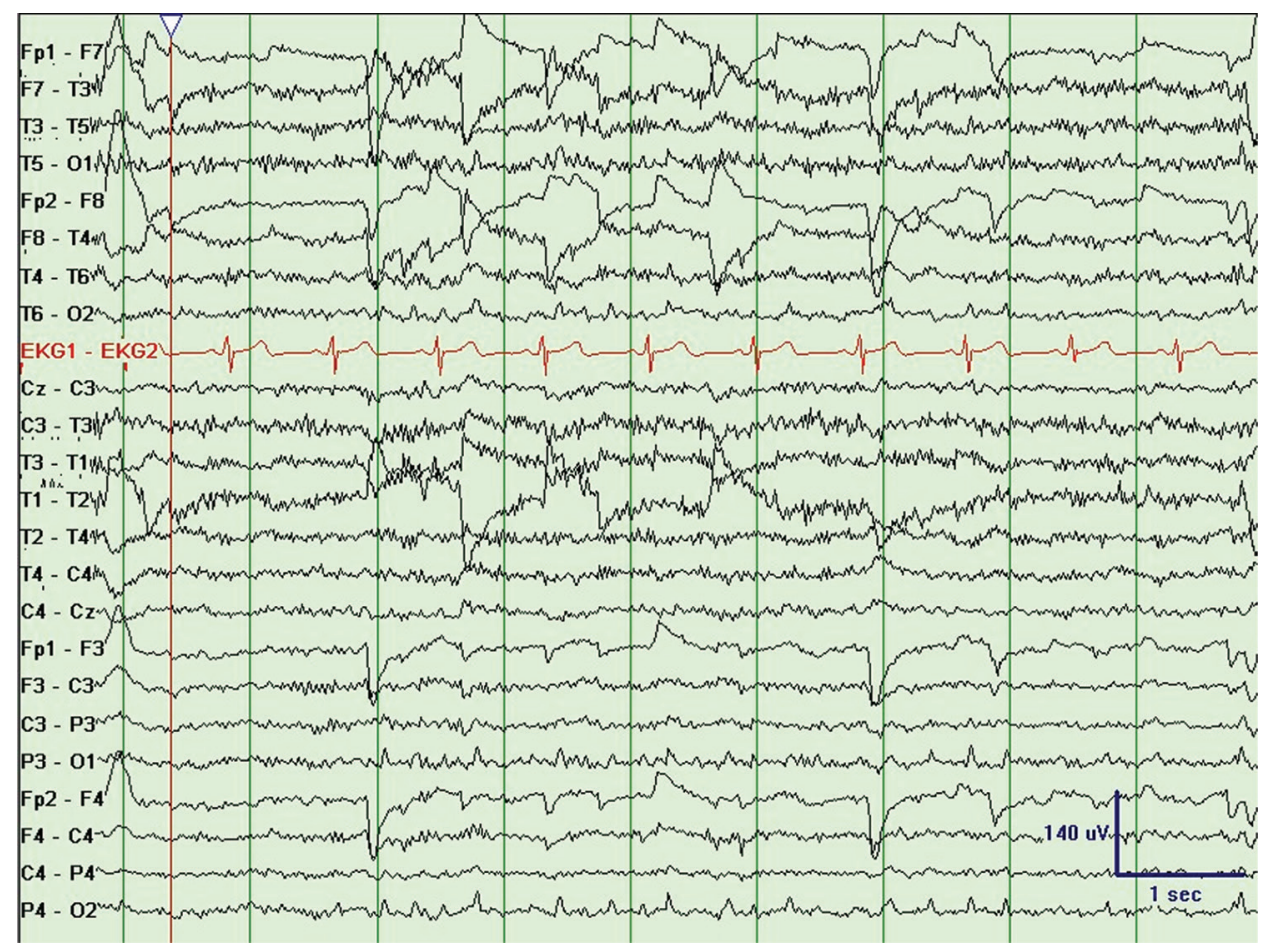

FIGURE 2.10. Bioccipital lambda waves in a 28-year-old with dizziness. Notice the frequent "scanning" eye movement artifact present in the F7 and T8 derivations.

ambda waves have been initially described as surface positive sharply contoured theta Lwaves appearing bilaterally in the occipital region. These potentials have a duration of 160 to $250 \mathrm{msec}$, and may at times be quite sharply contoured, asymmetrical, with higher amplitudes than the resting posterior dominant rhythm (Figure 2.10). When they may occur asymmetrically, they may be confused with IEDs, and potentially lead to the misinterpretation of the EEG. However, the positive polarity distinguishes lambda waves from occipital sharp waves with a deflection that is "up" instead of "down" given the end of chain localization. They are best observed in young adults when seen, though are more frequently found in children. Lambda waves are best elicited when the patient visually scans a textured or complex picture with fast saccadic eye movements. Placing a white sheet of paper in front of the individual will eliminate the visual input that is essential for their genesis and verify the relationship to rapid eye movements. 


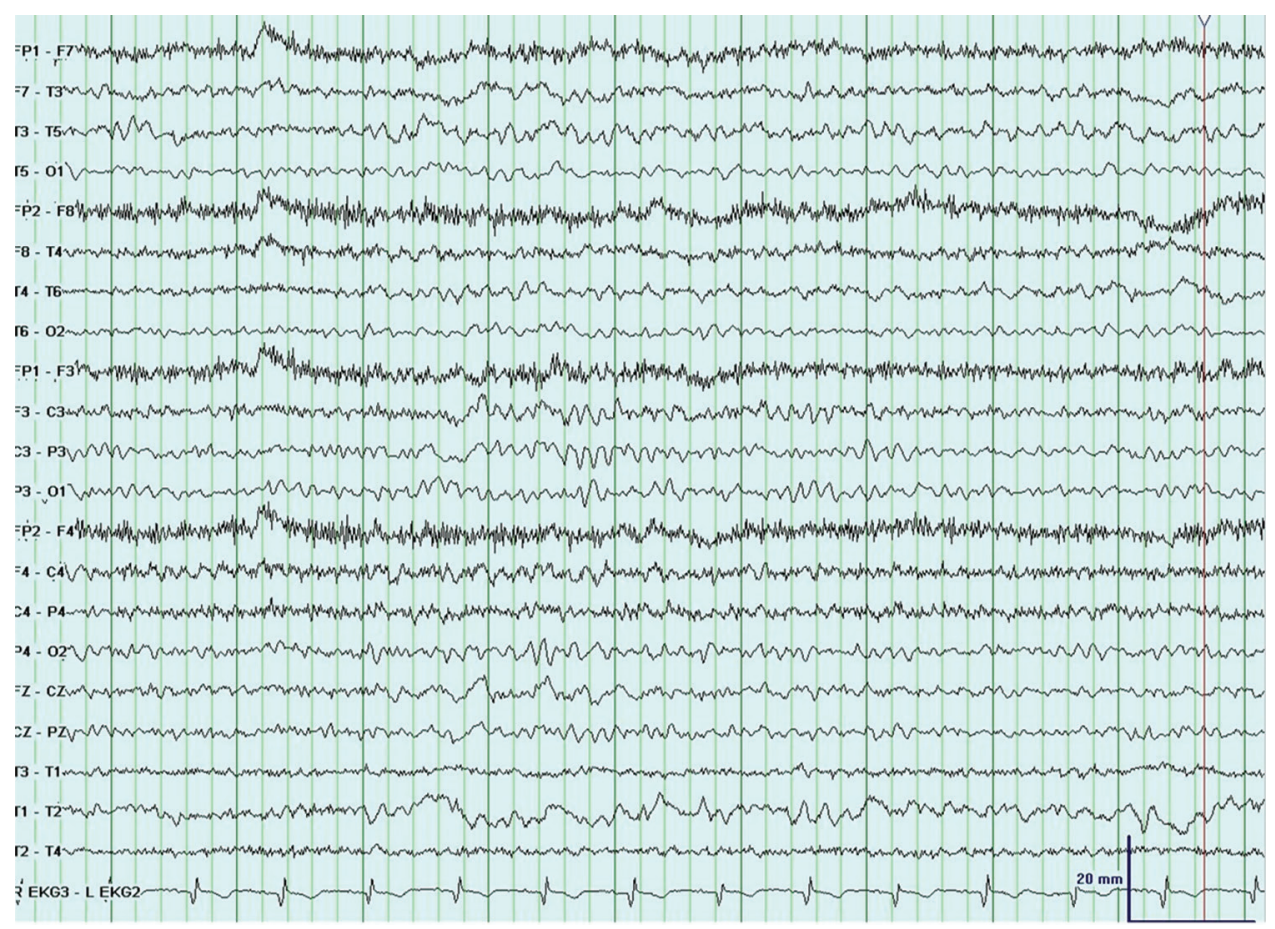

FIGURE 2.11. Intermittent left mid-temporal delta during transition to drowsiness in a normal 84-year-old evaluated for syncope.

$\mathrm{D}$ elta rhythms are frequencies that consist of $<4-\mathrm{Hz}$ activity. Delta comprises $<10 \%$ of the normal waking EEG by age 10 years. In the waking states, delta can be considered a normal finding in infancy and early childhood and in the elderly (Figure 2.11). The normal elderly may have rare irregular delta complexes in the temporal regions during the awake state. The appearance of delta is similar to the appearance of theta relative to spatial distribution, often left greater than right temporal head regions. However, delta is normally is present for $<1 \%$ of the recording. Some delta is normal in people older than 60 years, at the onset of drowsiness, in response to hyperventilation, and during slow wave sleep. Excessive generalized delta is abnormal and indicates an encephalopathy that is etiology nonspecific. Focal polymorphic delta usually indicates a structural lesion involving the white matter of the ipsilateral hemisphere, especially when it is continuous and unreactive. 


\section{NORMAL SLEEP ARCHITECTURE}

ormal drowsiness may be associated with state changes that manifest as paroxysmal bursts of theta frequencies. Non-REM stage 1 (N1) sleep is defined by the presence of vertex waves. These are $200 \mathrm{msec}$ diphasic sharp transients with maximal negativity at the vertex $(\mathrm{Cz})$ electrode during light sleep, though they may be seen in stage N1 to N3 sleep. They are bilateral, synchronous, symmetric, and may be induced by auditory stimuli. Vertex waves can appear spiky (especially in children) but should normally never be consistently lateralized. Other features include attenuation of the alpha rhythm, greater frontal prominence of beta, slow rolling eye movements, and vertex sharp transients. In addition, positive occipital sharp transients (POSTs) are another feature signifying N1 sleep. These are surface positive, bisynchronous physiologic sharp waves with voltage asymmetries that may occur over the occipital regions as single complexes or in repetitive burst that may be present in both stage 1 and 2 sleep (Figure 2.12A-B).

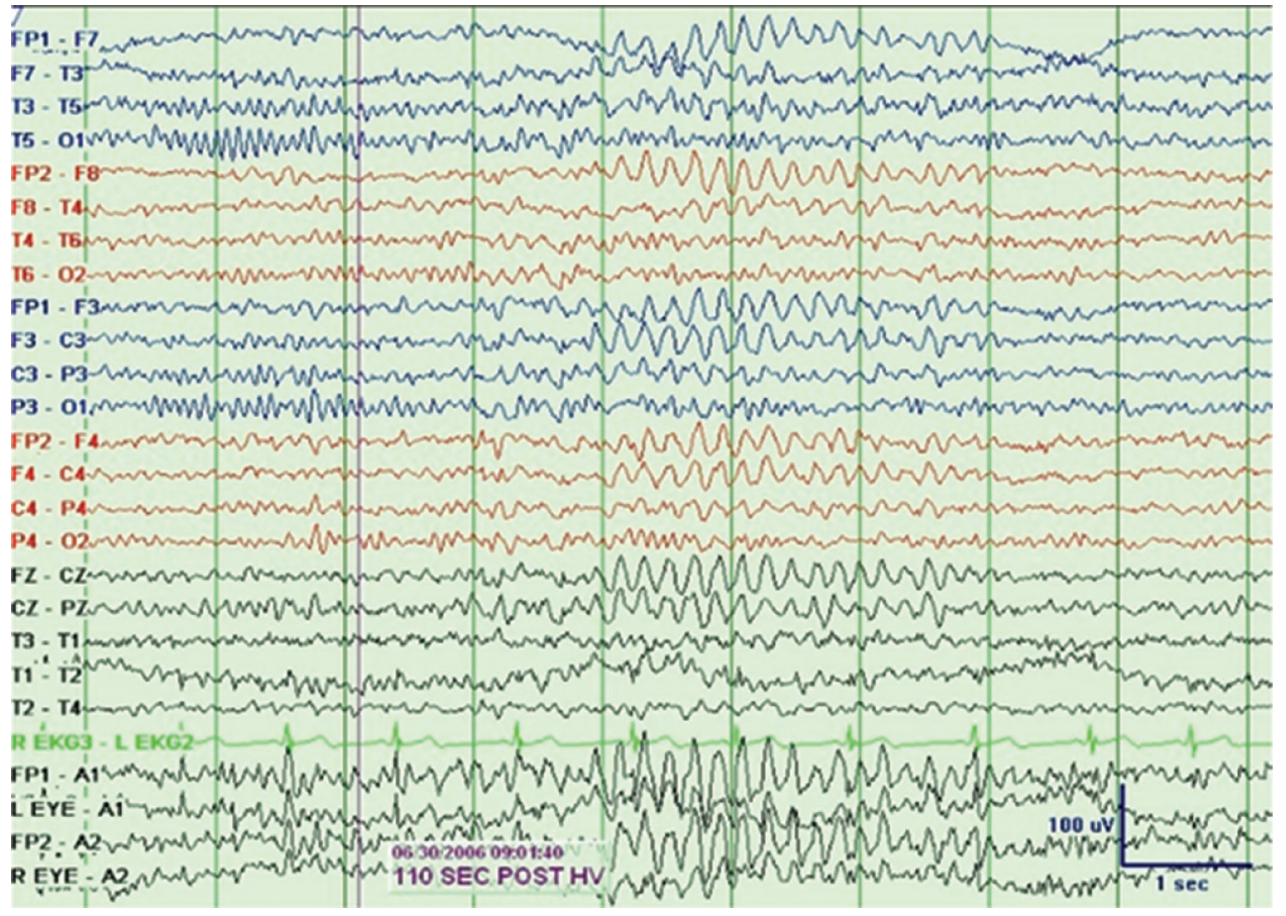

FIGURE 2.12A. Hypnogogic burst during transition to drowsiness and light sleep. (Continued) 


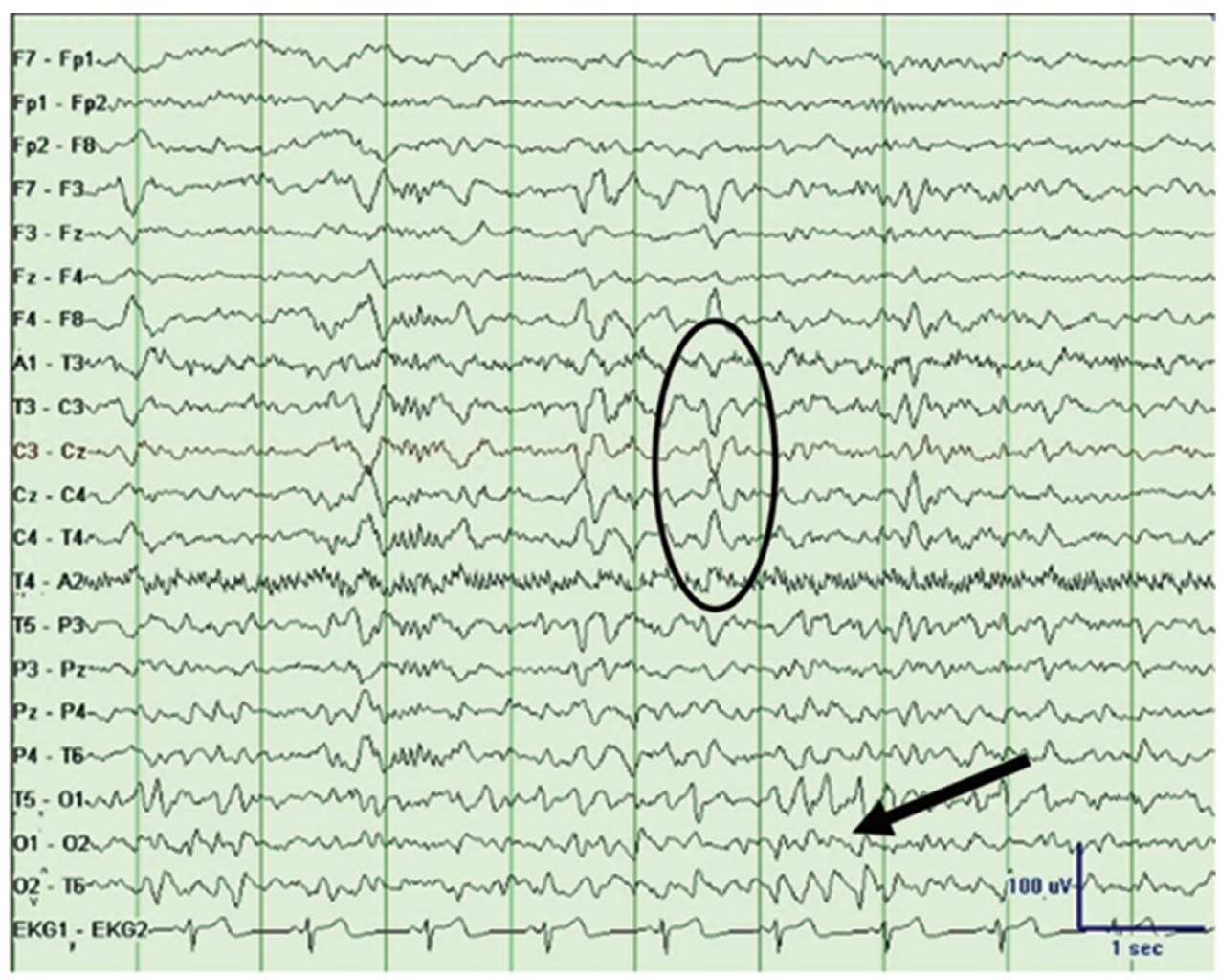

FIGURE 2.12B. Positive occipital sharp transients (POSTs) appearing in the lower three channels (arrow) in this bipolar circle montage demonstrating positive polarity (phase reversal) in the occipital region during N2 sleep. Notice the surface negative vertex waves signified by a $\mathrm{Cz}$ phase-reversal (oval). 


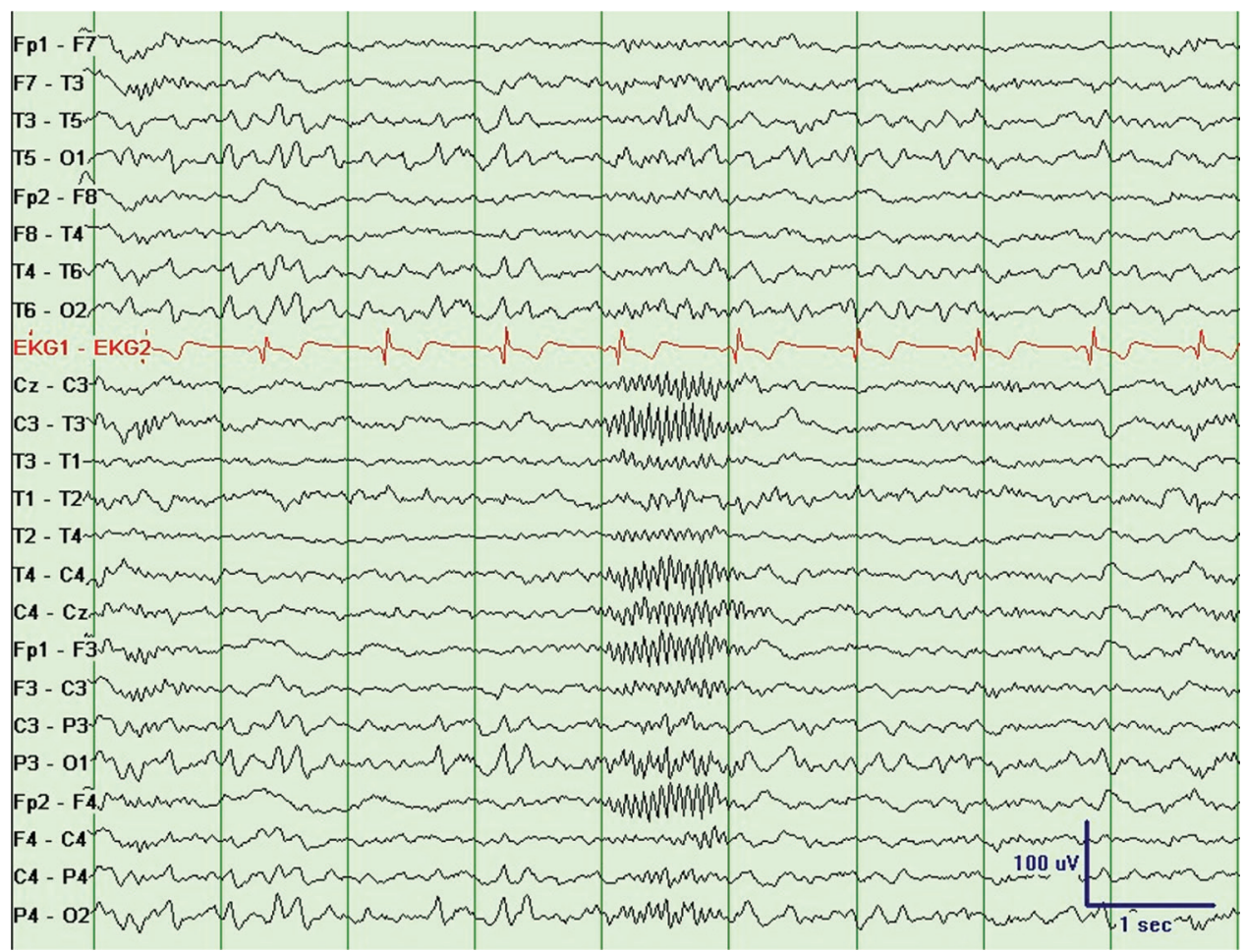

FIGURE 2.13. N2 sleep with prominent sleep spindles and positive occipital sharp transients (POSTs).

tage N2 sleep is defined by the presence of sleep spindles and $\mathrm{K}$ complexes. This stage has the same features as stage 1 with progressive slowing of background frequencies. Sleep spindles are transient, sinusoidal $12-$ to $14-\mathrm{Hz}$ activity with waxing and waning amplitude seen in the central regions with frontal representation by frequencies of 10 to $12 \mathrm{~Hz}$ (Figure 2.13). A K-complex is a high amplitude diphasic wave with an initial sharp transient followed by a high amplitude slow wave often associated with a sleep spindle in the frontocentral regions. A K-complex may be evoked by a sudden auditory stimulus. A persistent asymmetry of $>50 \%$ is abnormal on the side of reduction. 




FIGURE 2.14. N3 sleep depicting the slow delta waveforms that occupy majority of the background activity. Note the intermittent sharp morphology of positive occipital sharp transients (POSTs) in the 6th second.

3 or slow wave sleep best describes a deeper stage of non-REM sleep (a.k.a. deep sleep) and is comprised of 1.5 - to $2.5-\mathrm{Hz}$ delta frequencies occupying variable amounts of the background activity. Stage N3 sleep previously noted delta occupying $20 \%$ to $50 \%$ of the recording with voltages of $>75 \mu \mathrm{V}$, while stage $\mathrm{N} 4$ consisted of delta present for $>50 \%$ of the recording. Currently N3 sleep encompasses prior stages of sleep that were designated N3 and N4 sleep (Figure 2.14). 




FIGURE 2.15. REM sleep with horizontal rapid eye movement artifact on the EEG most apparent in the F7 and F8 derivations.

EM sleep is characterized by rapid eye movements (arrows), loss of muscle tone, and central sharply contoured theta waves (a.k.a. sawtooth waves) in the EEG (see Figure 2.15). Non-REM and REM sleep cycles alternate four to six times during a normal night's sleep. A predominance of non-REM appears in the first part of the night, while REM is longer and more widely represented in the last third of the night. A routine EEG with REM may reflect sleep deprivation and not necessarily a disorder of sleep-onset REM such as narcolepsy. 


\section{ACTIVATION PROCEDURES}

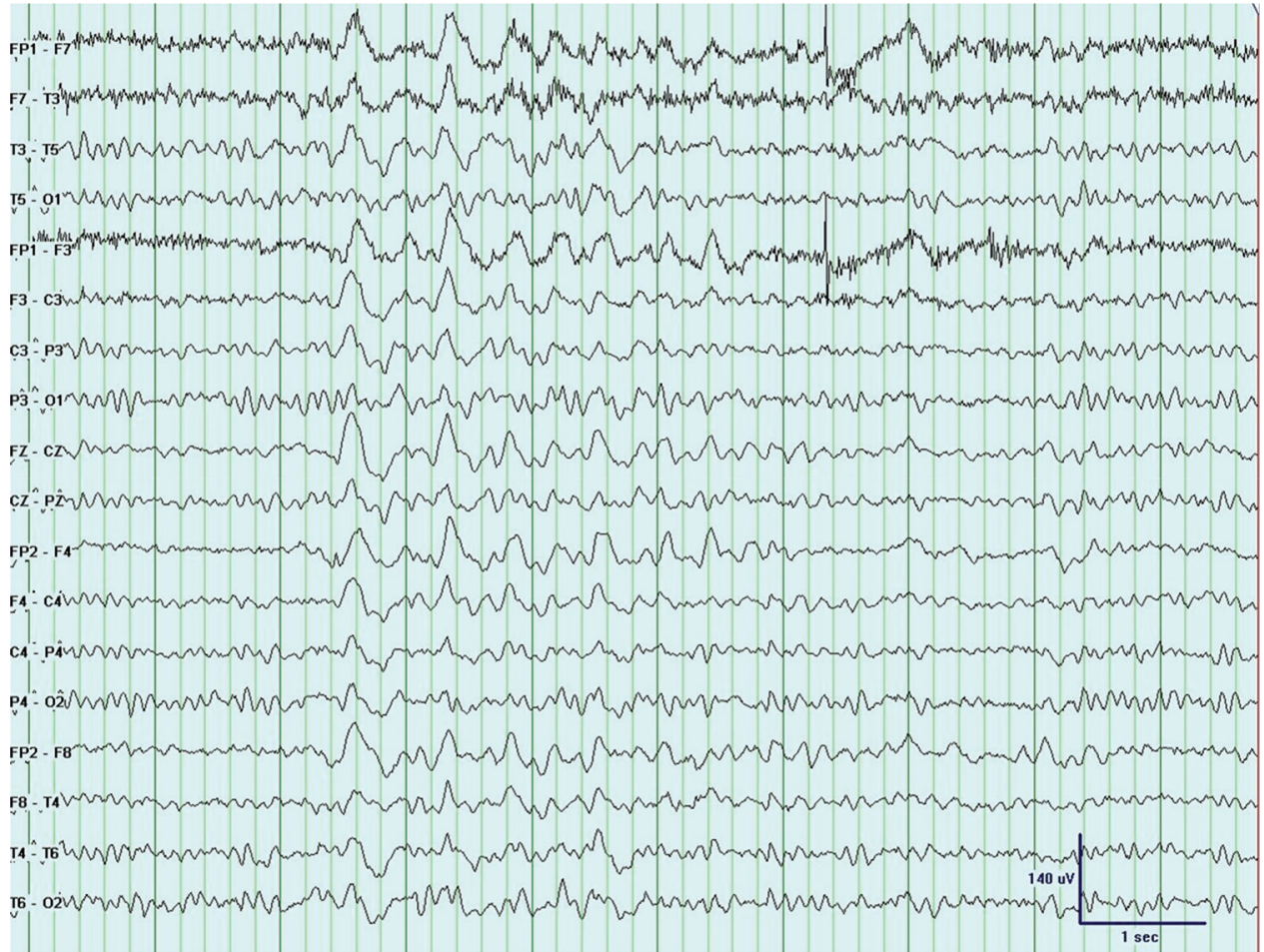

FIGURE 2.16. Normal buildup during hyperventilation.

Lyperventilation is routinely performed for 3 minutes in most EEG laboratories. The purpose is to create cerebral vasoconstriction through respiratory means promoting systemic hypocarbia. Hyperventilation normally produces a bilateral increase in theta and delta frequencies (buildup) that is frontally predominant, and often high amplitude (Figure 2.16). Resolution of the effect occurs normally within 1 minute. Activation, or the generation of epileptiform discharges, is infrequently seen in those with localizationrelated epilepsy $(<10 \%)$, however may approach $80 \%$ for those with generalized epilepsies that include absence seizures. Hyperventilation may produce focal slowing in patients with an underlying structural lesion. It should not be performed in patients with severe cardiac or pulmonary disease, acute or recent stroke, significant large vessel cerebrovascular disease, sickle cell anemia or trait, and should be used with caution during pregnancy. 




FIGURE 2.17A. Photic driving at $20 \mathrm{~Hz}$ seen in the P3-O1, P4-O2, T5-O1, and T6-O2 derivations.

(Continued)

Intermittent photic stimulation normally produces potentials exquisitely time-locked to the frequency of the intermittent light stimulus and is referred to as photic driving. Response depends upon background illumination and the distance of the light source from the patient. Distances of $<30 \mathrm{~cm}$ from the patient are used to optimize the effect of stimulation. Flashes are very brief and delivered in sequence from 1- to $30-\mathrm{Hz}$ flash frequencies for approximately 10 seconds before stopping the stimulus. Sub-harmonics and harmonics of the flash frequency may be seen. Photic driving is usually greatest in the occipital location, in frequencies approximating the alpha rhythm, when the eyes are closed (Figure 2.17A). Photomyoclonic (or photomyogenic) responses consist of frontally dominant muscle artifact that occurs when the flash evokes repetitive local contraction of the frontalis musculature (photomyogenic). The periocular muscles may also be affected with single lightening-like head jerks (photomyoclonic). Myogenic spikes occur 50 to $60 \mathrm{msec}$ after the flash and increase in amplitude as the stimulus frequency increases. The response is normal though may be seen in withdrawal syndromes or states of hyperexcitability. Similarly, the photoelectric response is an artifact composed of a train of brief, spiky potentials maximal in the frontopolar electrode derivations caused by a time-locked response to the light flash frequency (Figure 2.17B). 


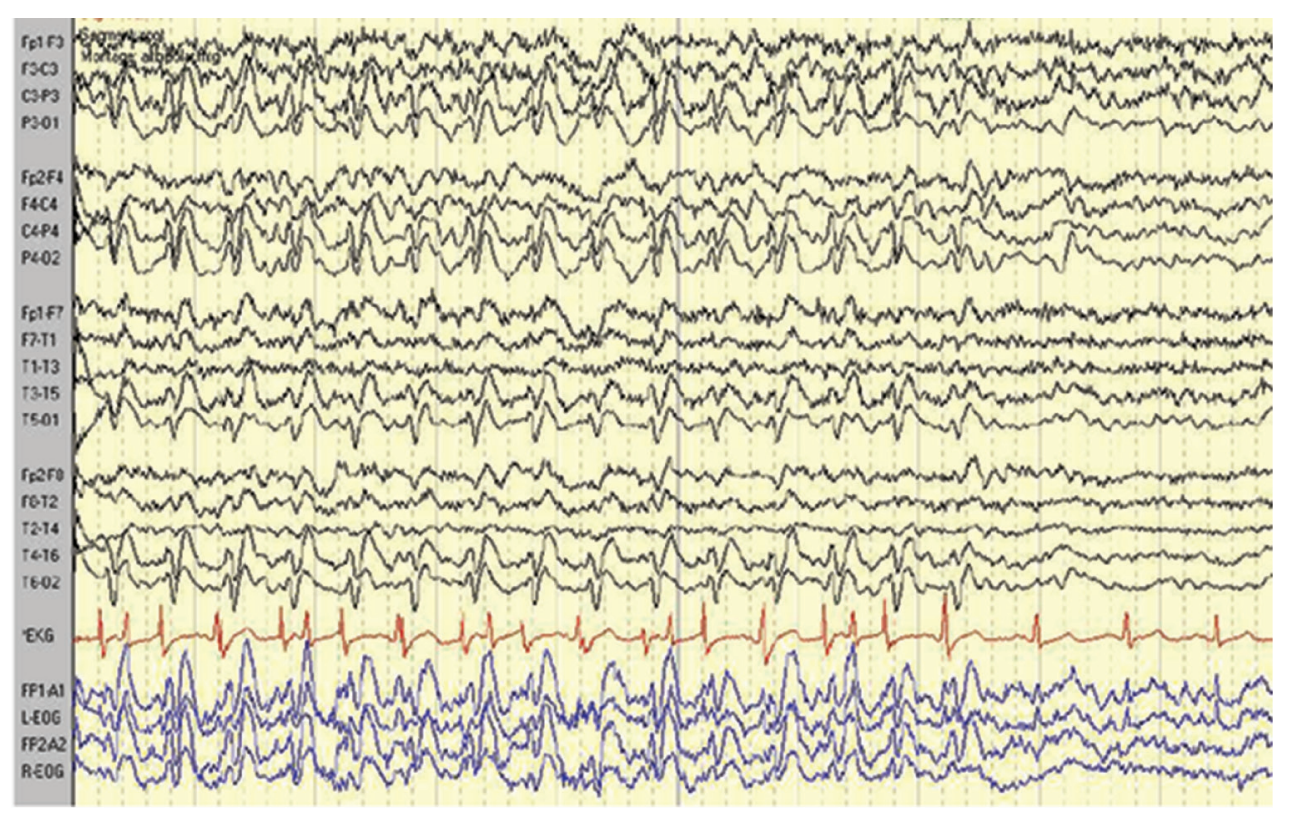

FIGURE 2.17B. Spike driving during intermittent photic stimulation at $2 \mathrm{~Hz}$. 


\section{BENIGN (NORMAL) VARIANTS}

ormal variations in background rhythms may cause confusion during interpretation of EEG. Phase reversals indicate location, not abnormality. Benign rhythmic and epileptiform waveforms may appear in the human EEG and are considered patterns of uncertain significance. Normal rhythms that appear as variations of normal, or epileptiform in morphology may serve as the basis for confusion and lead some to misinterpretation of the EEG. The rate of IEDs in standard EEG recordings obtained in nonepileptic individuals without progressive neurologic disorder has been reported as $0.5 \%$ to $2.6 \%$ in adults and $2.2 \%$ to $3.5 \%$ in children. Previously, many of these EEG patterns were felt to be associated with seizures, headaches, abdominal pain, and behavioral disturbances. However, many patterns serving as a pitfall to interpretation are considered benign variations, and do not possess significance specific for epileptic seizures.

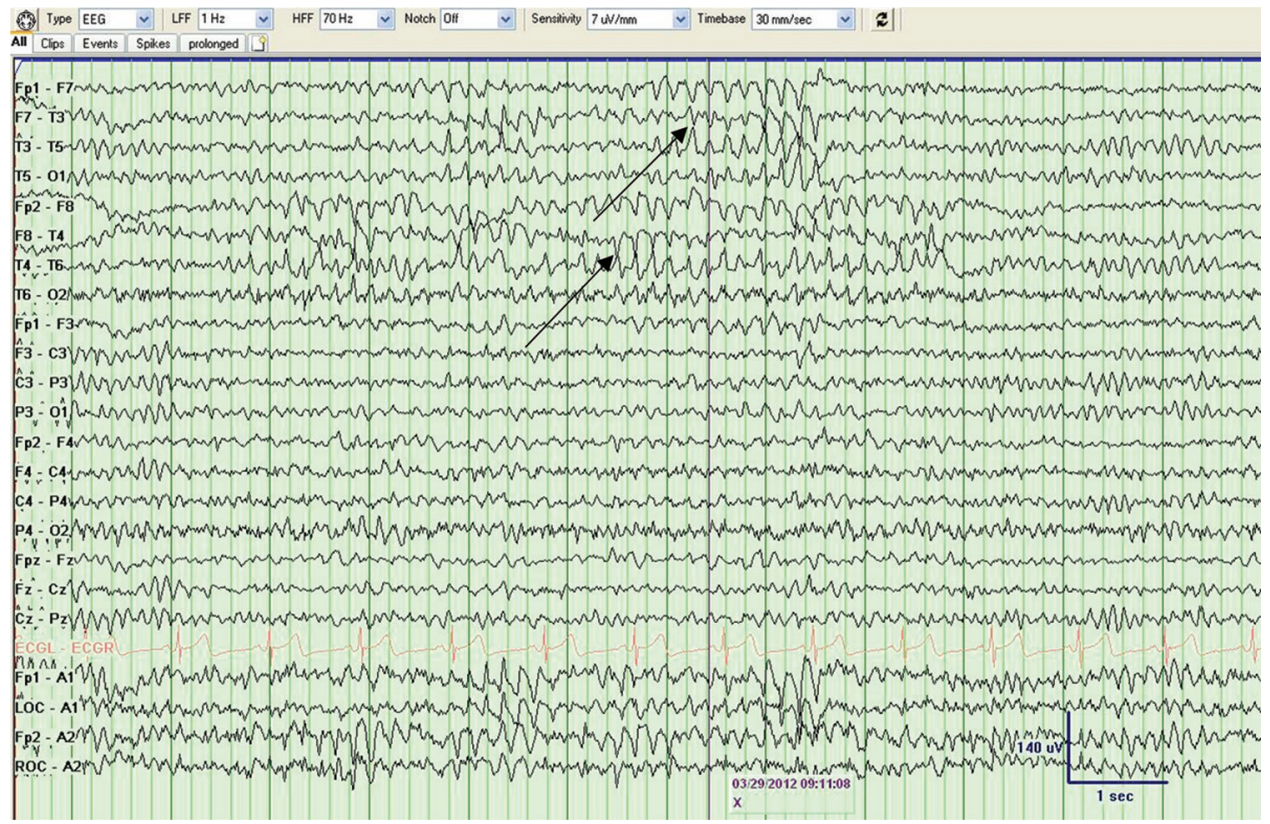

FIGURE 2.18. Rhythmic mid-temporal theta bursts in drowsiness (arrows). Note the sharply contoured morphology.

$\mathrm{R}^{\mathrm{h}}$ hythmic temporal theta bursts in drowsiness is the preferred term for what was formerly known as psychomotor variant. This pattern occurs in $0.5 \%$ to $2.0 \%$ of selected normal adults and consists of bursts or runs of 5- to 7- $\mathrm{Hz}$ theta waves that may appear sharp, flat, or notched in appearance. It is maximal in the mid-temporal derivations and was referred to as rhythmic mid-temporal theta bursts in drowsiness. It is an interictal pattern that does not evolve spatially or temporally, though may be represented bilaterally or independently over both hemispheres. It is seen in adolescents and adults in relaxed wakefulness (Figure 2.18). 


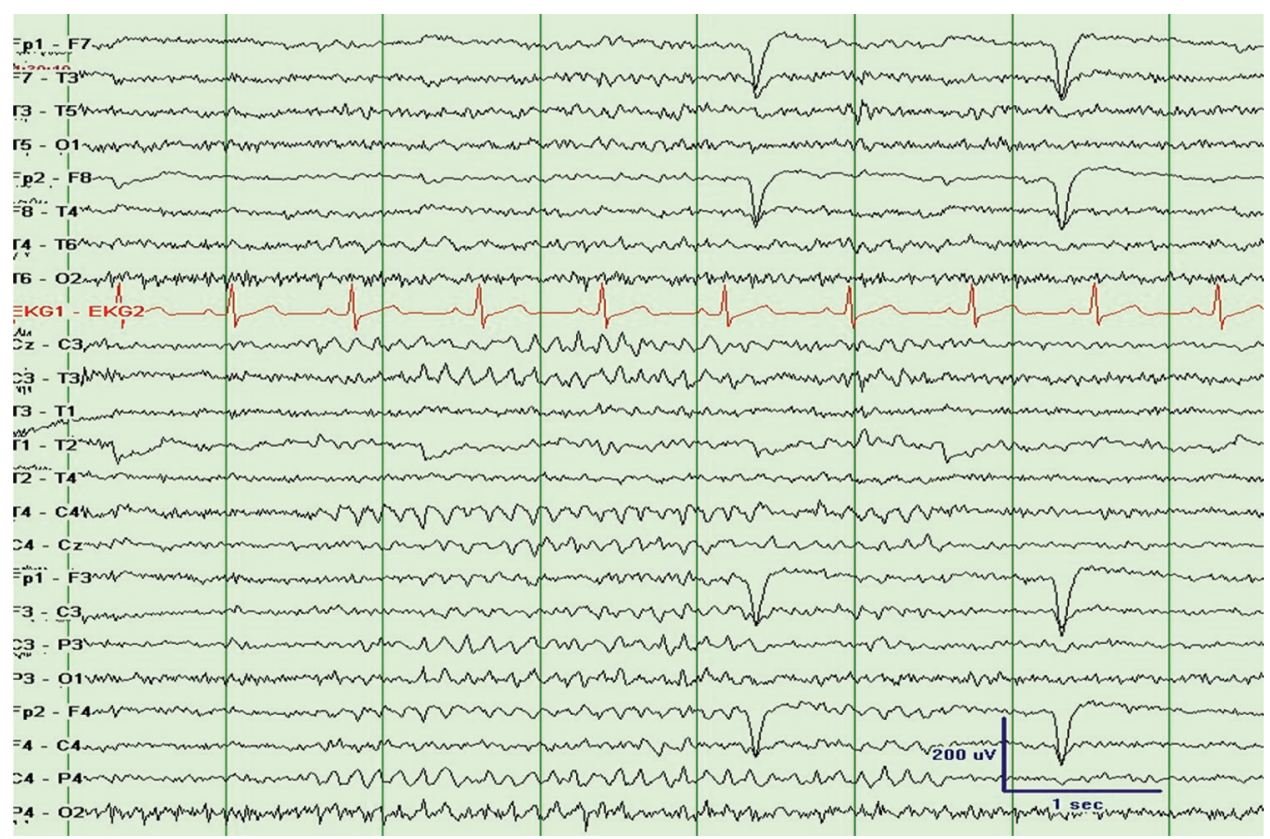

FIGURE 2.19. Central theta (maximal at $\mathrm{Cz}$ ) seen during the awake state in a 35-year-old with migraine headaches.

focal sinusoidal or arciform 4- to 7-Hz theta rhythm maximally expressed over the midline vertex region was first described by Ciganek (Figure 2.19). While morphologically it may resemble a $m u$ rhythm, it is not similarly reactive, is slower in frequency, and occurs both in drowsiness and the alert state. While initially felt to be a projected rhythm in temporal lobe epilepsy, it has been seen in a heterogeneous population and is therefore of nonspecific clinical significance unrelated to epilepsy. 


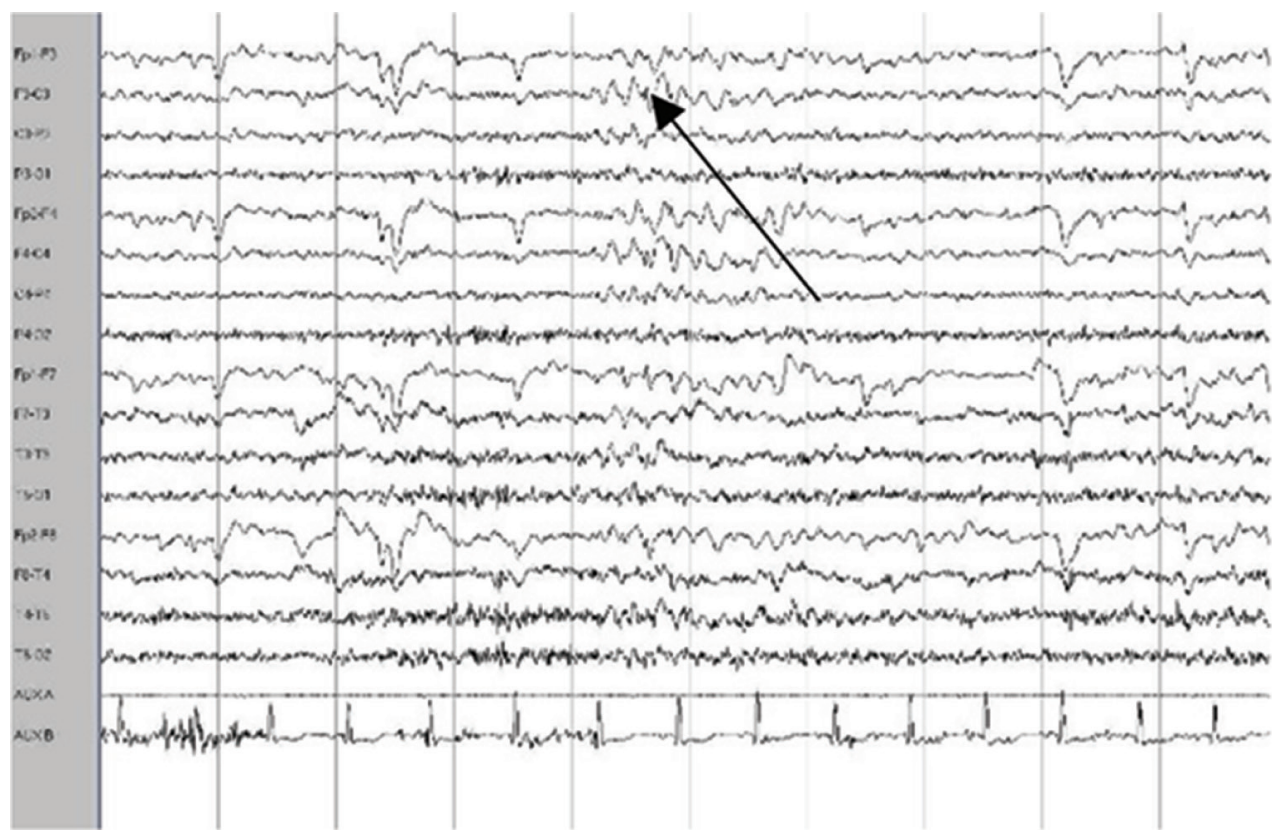

FIGURE 2.20A. Six-Hz spike-and-waves with frontal predominance. Note the appearance of a "phantom" low amplitude spike (arrow) as part of the wakefulness, high amplitude, anterior, male (WHAM) variant in the 5 th second of the EEG in an awake patient with temporal lobe epilepsy. (Continued)

—irst described by Walter in 1950, spike-and-wave discharges at $6 \mathrm{~Hz}$ were later coined - "phantom spike-and-wave" and separated into two waveforms by Hughes. These waveforms were referred to as "phantom spike wave" due their short duration and low amplitude spike. Initial descriptions included two forms. The acronyms WHAM (wakefulness, high amplitude, anterior, male) and FOLD (female, occipital, low amplitude, drowsy) were used to describe two primary subtypes. Bilateral, synchronous, 6-Hz spike-and-wave discharges may range from 5 to $7 \mathrm{~Hz}$, though with a typical repetition rate of $6 \mathrm{~Hz}$ lasting briefly for 1 to 2 seconds (Figure 2.20A). The spike is typically very low in amplitude, at times difficult to appreciate during routine interpretation of the EEG using visual analysis. When the spikes are of low amplitude and occur only during drowsiness, this usually represents a benign finding. Because they are seen in wakefulness and mild drowsiness and disappear in deep sleep, they can be distinguished from abnormal IEDs which increase during sleep. When they are seen with high amplitude spikes and occur with less than a $6-\mathrm{Hz}$ frequency or occur during wakefulness and persist into slow wave sleep, there is a greater association with seizures. FOLD is reported to be more benign ( $30 \%$ of epilepsy patients), while WHAM is more ictogenic (up to 90\%) (Figure 2.20B). 


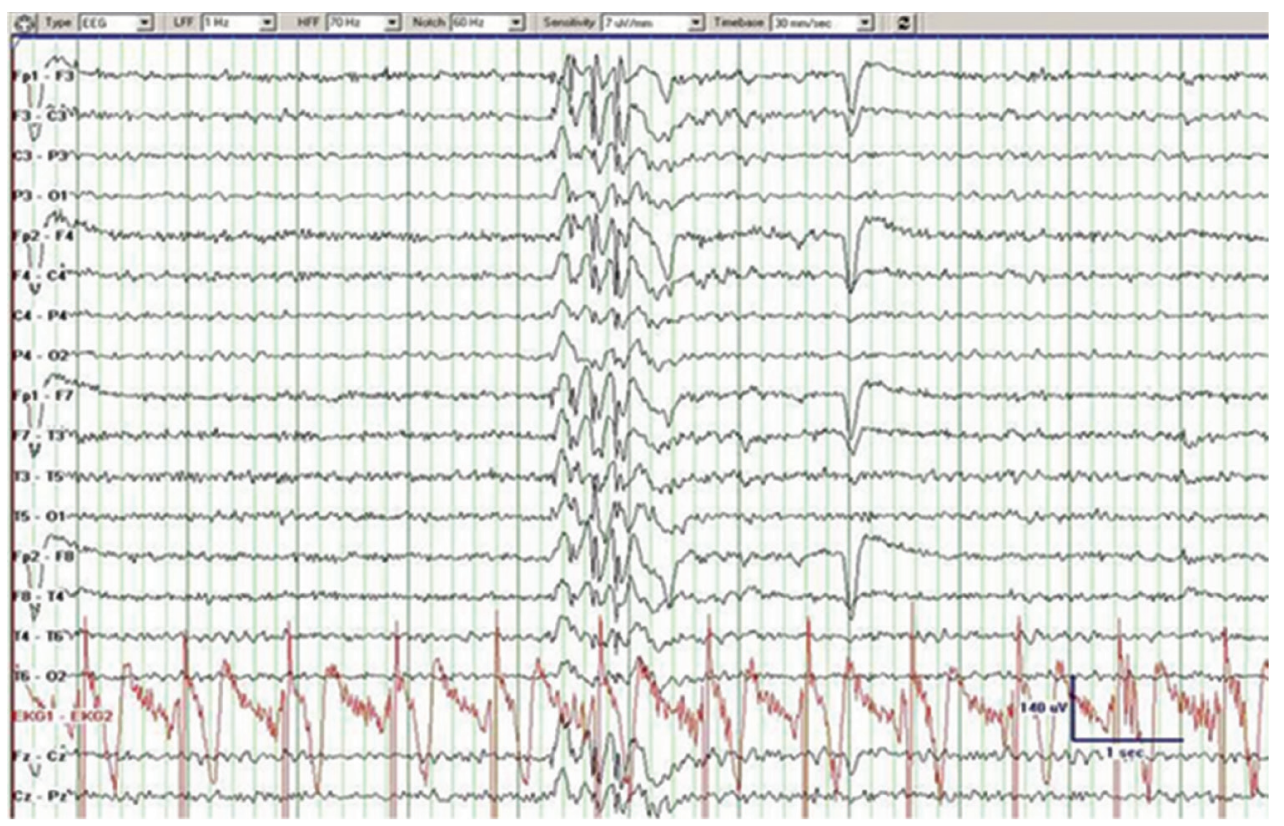

FIGURE 2.20B. In contrast note the $5.57-\mathrm{Hz}$ generalized spike-and-wave burst in a patient with juvenile myoclonic epilepsy. 


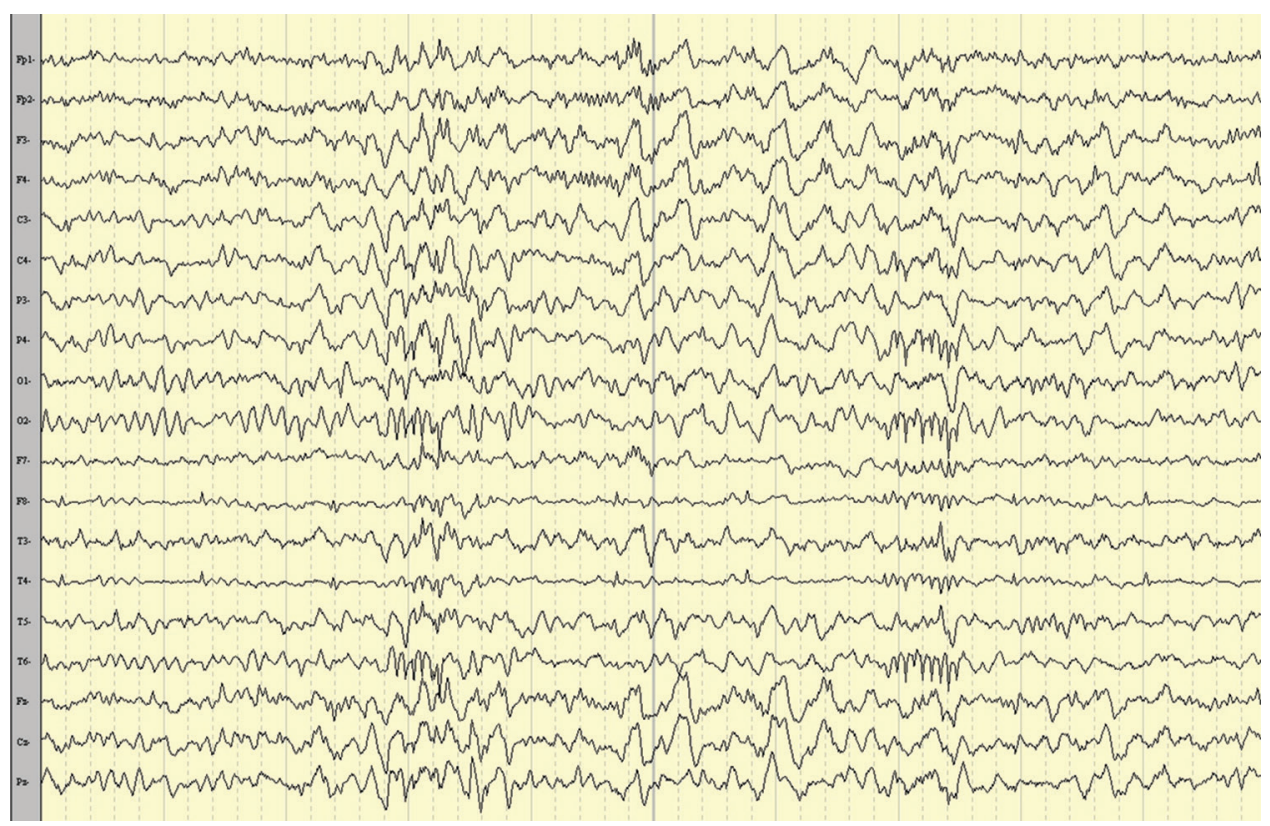

FIGURE 2.21. Fourteen- and 6-Hz positive bursts maximal in the T6 electrode derivation in a linked-ears reference montage. Note the downward deflection and prominent $14-\mathrm{Hz}$ frequency in this ear montage reflecting the positive polarity.

E ourteen- and 6-Hz positive bursts (originally called 14- and 6-Hz positive spikes) have also been called ctenoids. They appear in the EEG in bursts of positive comb-like spindles mainly over the posterior temporal head regions (Figure 2.21). They are present most frequently at a rate of $14-$ or $6-$ to $7-\mathrm{Hz}$ and last $1 / 2$ to 1 second in duration. The $14-\mathrm{Hz}$ frequency is most prevalent, and the $6-\mathrm{Hz}$ burst may appear with or without the faster frequencies. They are most common during adolescence, though they may persist into adulthood and decrease with age. The bursts are usually unilateral or bilaterally asynchronous with a shifting predominance involving one hemisphere to a greater degree. A contralateral ear reference montage and greater inter-electrode distance best demonstrate these bursts. 


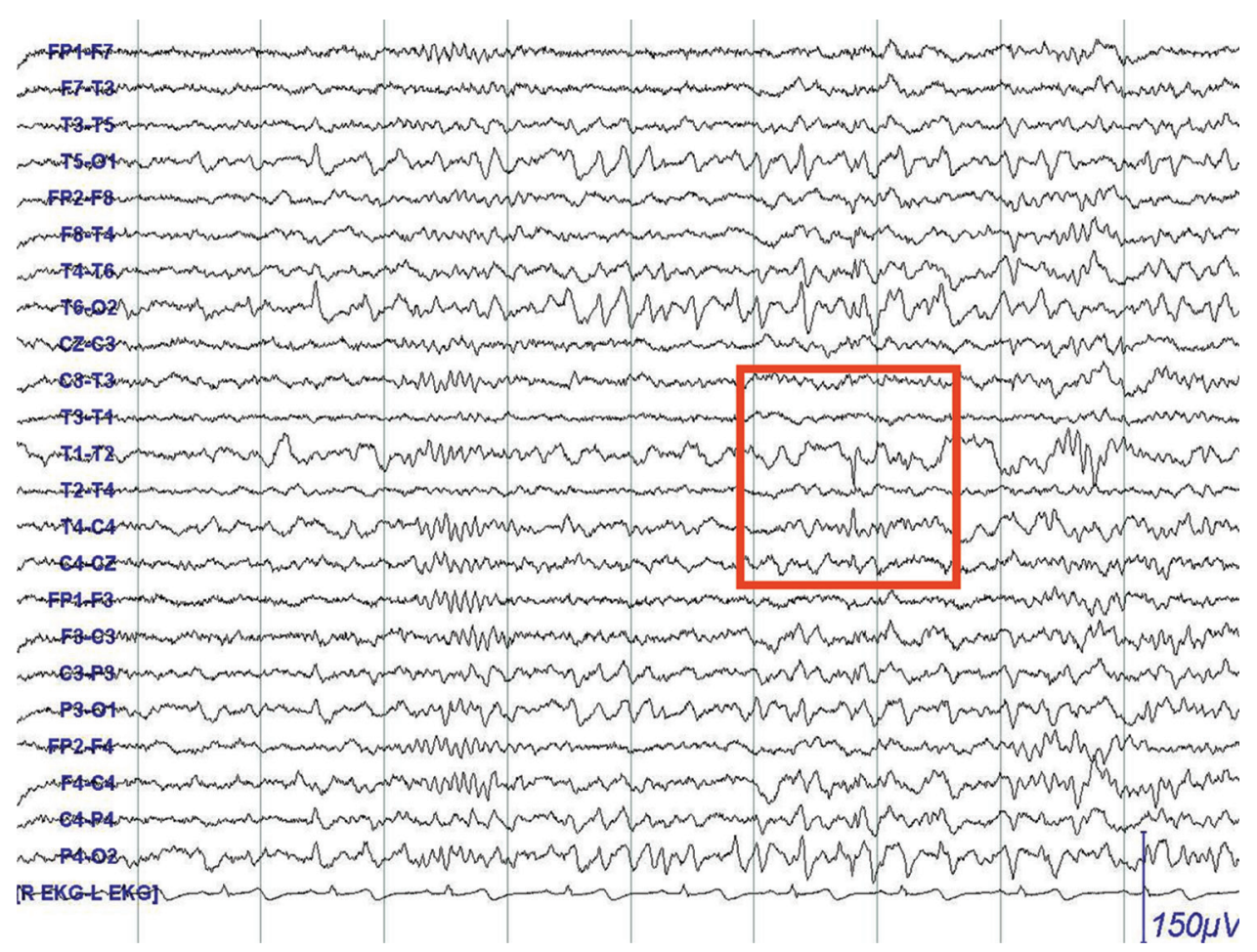

FIGURE 2.22. A right benign epileptiform transients of sleep (BETS) in the temporal region during stage 2 sleep. Note the higher amplitude in the T1-T2 channel with a longer inter-electrode distance.

D ifferent terms have been used to describe "small sharp spikes." These were also previously known as "benign sporadic sleep spikes" and now known as "benign epileptiform transients of sleep." These signals are comprised of low voltage $(<50 \mu \mathrm{V})$, brief duration $(<50 \mathrm{msec})$, simple waveform, with a monophasic or diphasic spike and broad hemispheric field of spatial distribution (Figure 2.22). This benign variant of uncertain significance has the morphology of a spike, though it has a rapidly ascending limb and steep descending limb best seen in the anterior to mid-temporal derivations during non-REM sleep. They are most common in adults. They may be $>50 \mu \mathrm{V}$, have a duration $>50 \mathrm{msec}$, and may appear with an after-going slow wave (usually of lower amplitude than the spike). They are not associated with focal slowing. They do not occur in runs. The most distinguishing characteristic is that they disappear in slow wave sleep. They appear as a unilateral discharge but are almost always independent when they are bilateral. They may possess a field that may correspond to an oblique transverse dipole resulting in opposite polarities over opposite hemispheres when they are bilateral. BETs occur during light sleep, maintain identical morphology, have a broad field, and have a variable after-going slow wave, while pathological spikes are state independent, have variable morphology, have a narrow field, and have slow waves of equal or higher amplitude. 


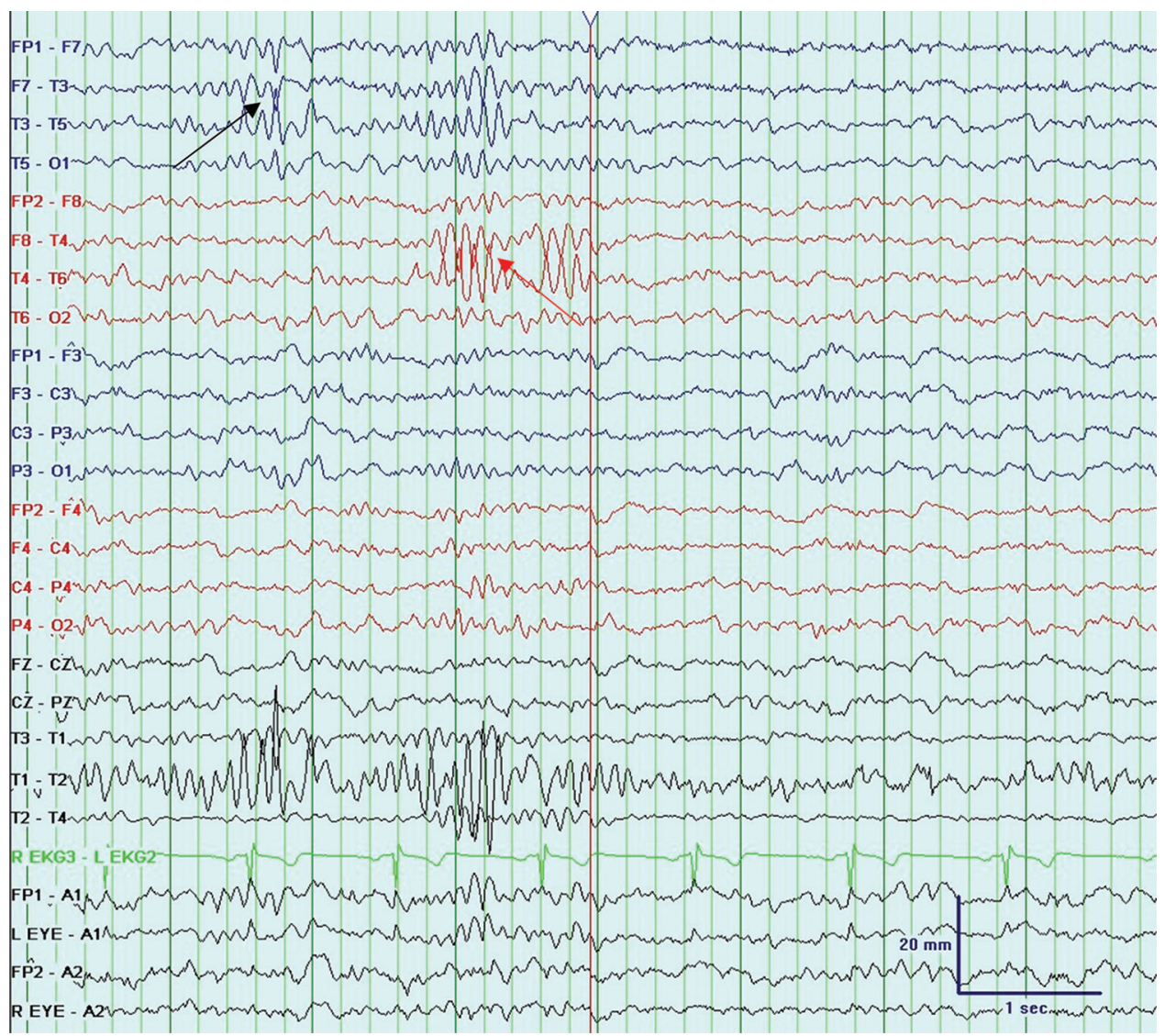

FIGURE 2.23. A burst of wicket waves maximal in the left (black arrow) and right (red arrow) mid-temporal derivations in this EEG.

ursts of wicket spikes are most common in adults $>30$ years of age. They occur within the $6-$ to $11-\mathrm{Hz}$ bandwidth and can obtain amplitudes of up to $200 \mu \mathrm{V}$. They are seen over the temporal regions during drowsiness and light sleep and are usually bilateral and independent. They typically occur in bursts, though may be confused with IEDs especially when they occur independently or as isolated waveforms (Figure 2.23). No focal slowing or after-going slow-wave component is seen and they likely represent fragmented temporal alpha activity. Similar frequency and morphology of a burst to the isolated waveforms is a means of providing support for the nonepileptogenic origin. While wicket spikes are considered an epileptiform normal variant, when selecting patients for EEG, they may appear concomitantly in the EEG of patients with epilepsy. 




\section{Onset}

FIGURE 2.24. Subclinical rhythmic electrographic discharge in adults (SREDA) in a 73-year-old during hyperventilation. No clinical signs were present. (Continued)

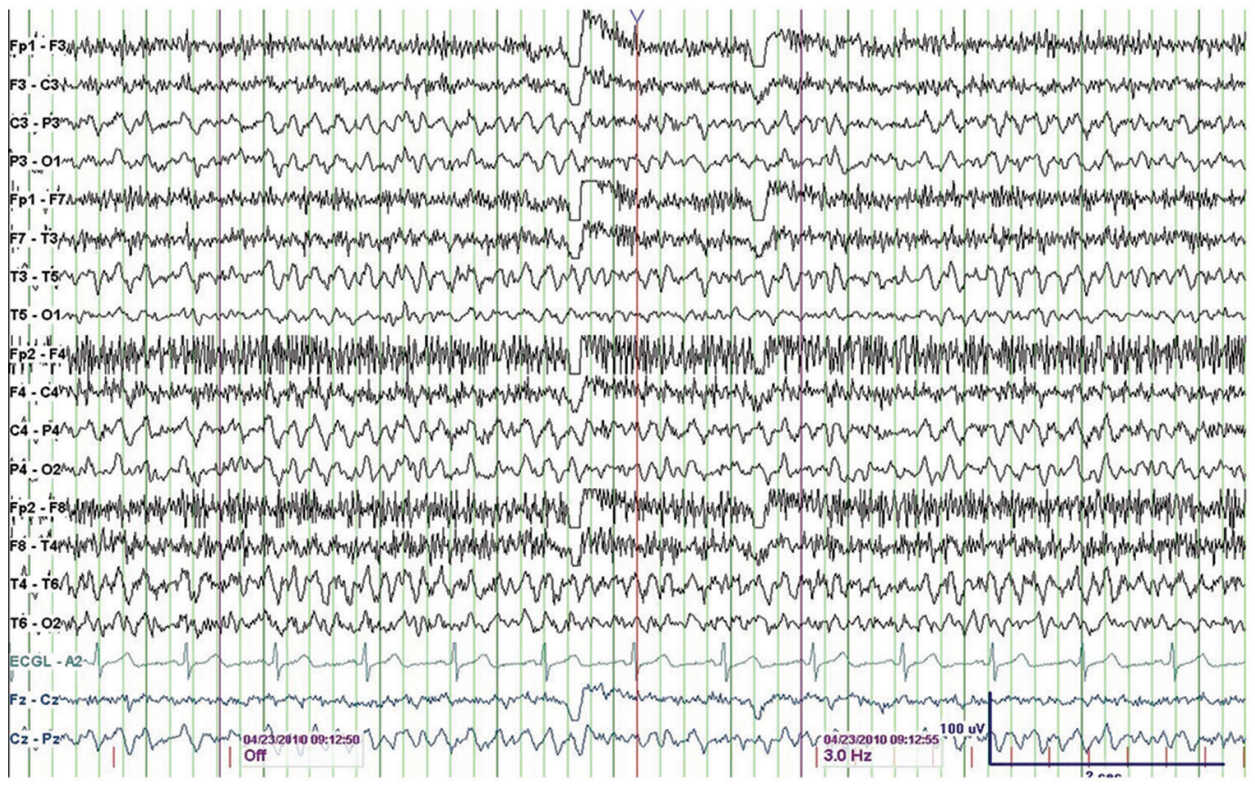

\section{Evolution}

FIGURE 2.24. (Continued) 


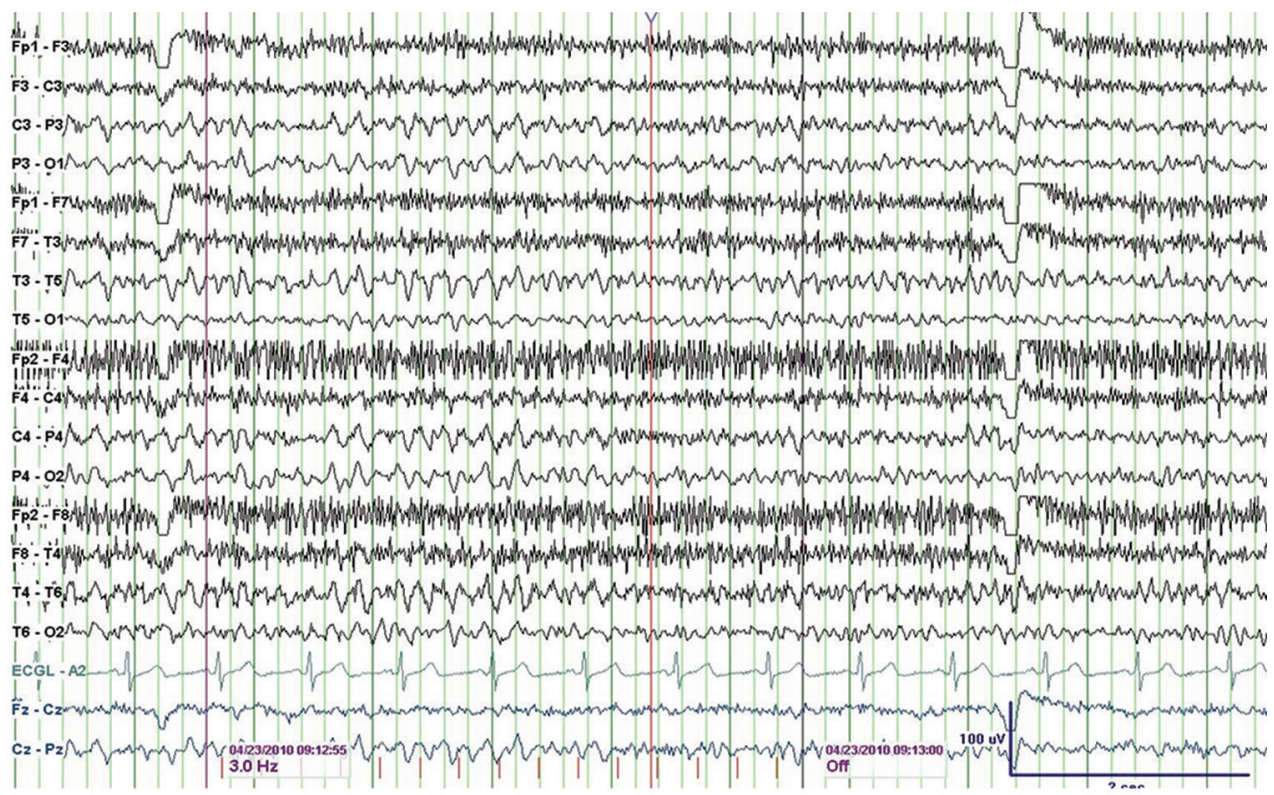

\section{Termination}

FIGURE 2.24. (Continued)

In contrast to many of the patterns of uncertain significance that mimic IEDs, subclinical rhythmic electrographic discharge in adults (SREDA) is a pattern that mimics the epileptiform characteristics of a subclinical seizure due to frequency evolution (Figure 2.24). SREDA is rare with an incidence of $0.06 \%$. No clinical features exist during it, either subjective or objective findings, and no association with epilepsy has been demonstrated. In contrast to most benign variants, SREDA is more likely to occur in those over 50 years of age and while the person is awake. The onset is abrupt, occurs at rest, and may be triggered by hyperventilation. SREDA appears as high-amplitude monophasic/diphasic sharp waves that coalesce to continuous delta pattern. It is bilateral, synchronous, symmetric, and often repeats in those $>50$ years. It may exist in two forms, either as a bilateral episodic burst of rhythmic sharply contoured 5- to $7-\mathrm{Hz}$ theta frequencies appearing maximal over the temporo-parietal derivations, or as an abrupt monophasic series of repetitive sharp or slow waveforms appear focally at the vertex recurring in progressively shorter intervals until a sustained burst is noted. It persists as $5-$ to $7-\mathrm{Hz}$ for 40 to 80 seconds $(40-100 \mu \mathrm{V})$. Bursts of SREDA usually last between 40 and 80 seconds and occur without postictal slowing. Rarely the two forms may appear in the same person (personal observation William O. Tatum.) 


\section{CONCLUSION}

Normal EEG lies at the foundation of interpretation to identify abnormal patterns. The qualitative analysis of EEG has an inherent subjectivity that may blur the boundaries between normal and abnormal. It is through training and experience that the ability to provide accurate identification of normal biorhythms, variations of normal waveforms, and benign variants of uncertain significance exists. By understanding normal waveform frequencies, morphologies, topography, amplitudes, locations, synchrony, and periodicity, physiological electrocerebral activity including sleep architectures are accurately able to be identified by the interpreter. Some epileptiform variants may be subject to misinterpretation and lead to inaccurate management of patients. It is critical to remain conservative as an interpreter to minimize the likelihood of imparting a misdiagnosis of patients to avoid overly aggressive treatment. With new waveforms still being identified, EEG continues to remain at the forefront of neurological evaluations as well as general medical conditions that involve brain function.

\section{ADDITIONAL RESOURCES}

American Clinical Neurophysiology Society. Guidelines and Consensus Statements. 2016. https:// www.acns.org/practice/guidelines

Benbadis SR, Tatum WO. Overinterpretation of EEGs and misdiagnosis of epilepsy. J Clin Neurophysiol. 2003;20:42-44. https://doi.org/10.1097/00004691-200302000-00005

Kural MA, Duez L, Hansen VS, et al. Criteria for defining interictal epileptiform discharges in EEG: a clinical validation study. Neurology. 2020;94:e2139-e2147. https://doi.org/10.1212/WNL. 0000000000009439

Markand, ON. Pearls, perils, and pitfalls in the use of the electroencephalogram. Semin Neurol. 2003;23(1):7-46. https://doi.org/10.1055/s-2003-40750

Maulsby RL. Some guidelines for assessment of spikes and sharp waves in EEG tracings. Am J EEG Technol. 1971;11:3-16. https://doi.org/10.1080/00029238.1971.11080808

Olejniczak P. Neurophysiologic basis of EEG. J Clin Neurophysiol. 2006;23(3):186-189. https://doi. org/10.1097/01.wnp.0000220079.61973.6c

Santoshkumar B, Chong JJ, Blume WT, et al. Prevalence of benign variants. Clin Neurophysiol. 2009;120(5):856-861. https://doi.org/10.1016/j.clinph.2009.03.005

Tatum WO. Normal "suspicious" EEG. Neurology. 2013;80(1 suppl 1):S4-S11. https://doi.org/10.1212/ WNL.0b013e31827974df

Tatum WO IV, Husain A, Benbadis SR, Kaplan PW. Normal human adult EEG and normal variants. J Clin Neurophysiol. 2006;23(3):194-207. https://doi.org/10.1097/01.wnp.0000220110.92126.a6

Westmoreland BF. Epileptiform electroencephalographic patterns. Mayo Clin Proc. 1996;71:501-511. https://doi.org/10.4065/71.5.501 